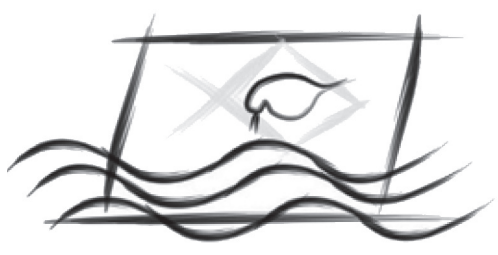

ECOTOX - Brazil
J. Braz. Soc. Ecotoxicol., v. 6, n. 1, 2011, 1-7

doi: 10.5132/jbse.2011.01.001

\title{
A Escolha do Anfípodo Bentônico para Ensaios Ecotoxicológicos com Sedimentos Marinhos
}

\author{
E. Bertoletti ${ }^{1}$ \\ ${ }^{1}$ Consultor independente, Rua Guaporé, 290, CEP 01109-030, São Paulo - SP
}

(Received May 6, 2010; Accepted August 4, 2010)

\begin{abstract}
RESUMO
Recentemente foi publicada a norma ABNT-NBR 15638 que descreve o método de ensaio ecotoxicológico com sedimento integral para os anfípodos bentônicos Leptocheirus plumulosus e Tiburonella viscana, os quais se constituem nos organismosteste mais utilizados com esse tipo de amostra em estudos brasileiros de ambientes marinhos ou estuarinos. Com o objetivo de analisar algumas condições nos ensaios com esses dois anfípodos, foram compilados os dados de um monitoramento ambiental efetuado na região oceânica de Santos-SP. Os resultados dos ensaios ecotoxicológicos com Leptocheirus plumulosus e Tiburonella viscana indicaram que os sedimentos da região estudada estão pouco contaminados. A análise da precisão das replicatas utilizadas nos ensaios revela que o coeficiente de variação foi idêntico (C.V.=22,0\%) para ambos os anfípodos. No entanto, no ensaio com Tiburonella viscana houve uma fraca explicação para a correlação desse coeficiente com a intensidade do efeito tóxico. As análises de regressão indicaram haver uma correlação entre a intensidade do efeito tóxico para Tiburonella viscana e a concentração de N-Kjeldahl e, também, a proporção de silte+argila nos sedimentos, fato que não ocorreu com Leptocheirus plumulosus. Com base nesse e em outros estudos foi possível estimar que a sobrevivência de Tiburonella viscana é afetada em sedimentos pouco contaminados que contenham proporções de silte+argila (finos) maiores que 33\%.
\end{abstract}

Palavras-chave: Anfípodo, Leptocheirus plumulosus, Sedimento, Silte+argila, Tiburonella viscana.

\section{ABSTRACT \\ The Choice of Benthic Amphipod for Ecotoxicological Tests with Marine Sediments}

Recently, the ABNT-NBR 15638 standard was published normalizing the method of ecotoxicological test with whole sediment using the benthic amphipods Leptocheirus plumulosus and Tiburonella viscana. These test organisms are the most widely used for this matrix in studies of Brazilian marine and estuarine environments. In order to analyse some experimental conditions in toxicity tests with these two amphipods, data from a monitoring program conducted in the oceanic region of Santos-SP were compiled. The results of ecotoxicological tests with Leptocheirus plumulosus and Tiburonella viscana indicated that sediments in the region have low contamination levels. The precision within replicates used in the tests showed that the coefficient of variation was similar $(\mathrm{CV}=22 \%)$ for both amphipods, however, there was a weak explanation with the intensity of the toxic effect in the Tiburonella viscana test. Regression analysis indicated significant correlation among the intensity of the toxic effect to Tiburonella viscana and the concentration of N-Kjeldahl, and also the proportion of silt+clay in sediments, which did not occur to Leptocheirus plumulosus. Based on present study and others, it was possible to estimate that survival of Tiburonella viscana is affected in sediments with low levels of contamination, but more than $33 \%$ of silt+clay (fines).

Keywords: Amphipod, Leptocheirus plumulosus, Sediment, Silt+clay, Tiburonella viscana.

* Corresponding author: Eduardo Bertoletti, e-mail: ebertoletti@ terra.com.br. 


\section{INTRODUÇÃO}

No Estado de São Paulo as operações de dragagens nas áreas portuárias de Santos têm demandado, desde 1998, o uso de ensaios ecotoxicológicos, especialmente para avaliar os potenciais danos de sedimentos contaminados de ambientes marinhos ou estuarinos e, também, para verificar o cumprimento de exigências da Resolução CONAMA 344 (CONAMA, 2004).

Segundo Prósperi \& Nascimento (2008), no Brasil, diversos organismos aquáticos têm sido utilizados nos ensaios ecotoxicológicos com sedimento estuarino/marinho, como por exemplo, os ouriços-do-mar Lytechinus variegatus e Echinometra lucunter, a ostra Crassostrea rhizophorae, o tanaidáceo Kalliapseudes schubarti, os anfípodos Tiburonella viscana e Leptocheirus plumulosus. Além disso, diferentes fases do sedimento são empregadas na exposição dos organismos durante os ensaios, tal como: a água intersticial, o elutriato, a interface água-sedimento, e o sedimento integral.

Devido a essa variedade experimental, não raro, os pesquisadores brasileiros direcionam seus estudos com sedimentos pela utilização das condições laboratoriais disponíveis, entretanto sem efetuar uma análise crítica prévia da representatividade ambiental dos ensaios ecotoxicológicos a serem executados. Como consequência, diagnósticos ou monitoramentos ambientais produzem resultados inesperados ou ilógicos, além de serem comparados indevidamente com os resultados de outros estudos.

Certamente as escolhas indevidas também ocorreram em outros países, no entanto, debates técnicos auxiliaram na escolha dos ensaios apropriados. Por exemplo, em discussão abrangente sobre a avaliação de risco de sedimentos contaminados pesquisadores americanos consideraram, por consenso, que os ensaios ecotoxicológicos que utilizam o sedimento integral e organismos bentônicos são aqueles de maior relevância ecológica (Ingersoll et al., 1997). Ao mesmo tempo, esses pesquisadores consideraram que os ensaios com elutriato não representam a medida real das condições dos sedimentos, sendo restritos apenas para avaliar o potencial de liberação de contaminantes do sedimento para a coluna d'água.

Com base nessas constatações, e no uso crescente das análises ecotoxicológicas em ambientes marinhos, pesquisadores brasileiros elaboraram a norma técnica ABNT-NBR 15638 (ABNT, 2008), a qual descreve o método de ensaio com sedimento integral e anfípodos bentônicos de águas costeiras. Assim, a referida norma aborda tanto o procedimento analítico de maior relevância ecológica bem como os dois organismos-teste mais utilizados em ensaios com sedimentos no Brasil, isto é, Leptocheirus plumulosus e Tiburonella viscana. Portanto, o presente estudo visa analisar algumas condições experimentais, em ensaios simultâneos com Leptocheirus plumulosus e Tiburonella viscana, de modo a fornecer informações para a escolha do organismo-teste nos ensaios ecotoxicológicos com sedimento integral.

\section{MATERIAL E MÉTODOS}

As informações apresentadas no presente artigo são originárias dos processos SMA-13740/04 e SMA-13781/02, os quais fazem parte de licenças de operação de dragagens de manutenção na região portuária de Santos-SP, no âmbito da Secretaria de Meio Ambiente do Estado de São Paulo. Essas informações fazem parte de relatórios mensais do monitoramento da área de abrangência de disposição oceânica de material dragado, em nove pontos de amostragem, conforme ilustrado na Figura 1.

Os pontos de amostragem estão identificados em numeração crescente, de acordo com a direção preferencial da pluma de dispersão dos sedimentos, isto é, sudeste-nordeste alinhada com a costa a partir do ponto 3 (área de descarte do material dragado).

Os ensaios ecotoxicológicos com Leptocheirus plumulosus e Tiburonella viscana foram realizados em dois laboratórios especializados, cada qual com um dos organismos-teste, que receberam amostras idênticas de sedimento. As amostras foram coletadas no período de maio/2007 a julho/2008. Do total de 126 ensaios ecotoxicológicos para cada organismo-teste, foram selecionados 108 resultados analíticos, os quais foram iniciados com defasagem menor que 30 dias entre os dois laboratórios, e que apresentaram qualidade analítica satisfatória (concentração de amônia apropriada, mortalidade no controle $\leq 15 \%$, e ensaio de sensibilidade com resultado dentro dos limites da carta-controle pré-estabelecida), de acordo com os métodos utilizados. Os ensaios com Leptocheirus plumulosus foram efetuados segundo USEPA (1994), enquanto os ensaios com Tiburonella viscana seguiram o descrito em Melo \& Abessa (2002). Resumidamente, ambos os métodos de ensaios consistiram da exposição de 10 organismos em cada uma das replicatas (4 ou 5), as quais continham $175 \mathrm{~mL}$ de sedimento e $750 \mathrm{~mL}$ de água marinha, sendo que os ensaios duraram 10 dias. A sensibilidade dos anfípodos foi verificada, mensalmente, por meio de cartas-controle previamente elaboradas pelos laboratórios.

Os sedimentos foram classificados quanto à granulometria em areia $(>0,063$ a $2,0 \mathrm{~mm})$, silte $(0,004$ a $0,063 \mathrm{~mm})$ e argila $(<0,004 \mathrm{~mm})$. As análises de carbono orgânico total e nitrogênio Kjeldahl foram efetuadas nas amostras de sedimento. As análises de regressão utilizadas, tanto lineares quanto múltipla, foram efetuadas utilizando-se o programa computadorizado Microcal Origin, versão 4.1, 16 bit (Northampton, MA/USA).

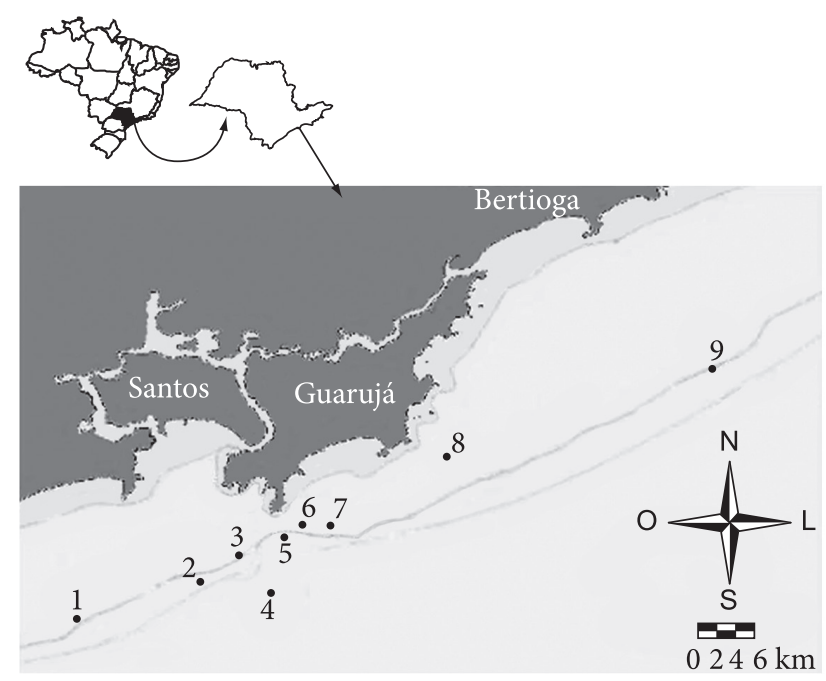

Figura 1 - Pontos de amostragem de sedimentos (numeração crescente de acordo com a direção preferencial da pluma de dispersão dos sedimentos). 


\section{RESULTADOS E DISCUSSÃO}

Os resultados das análises ecotoxicológicas, de granulometria, carbono orgânico total e nitrogênio Kjeldahl são apresentados na Tabela 1. Devido ao extenso número de amostras (108) de sedimento analisadas os resultados são apresentados em termos das médias e amplitudes das variáveis analisadas. A apresentação dos valores médios é conveniente por, resumidamente, representar melhor as características dos pontos de amostragem ao longo do tempo. Deve-se ressaltar, também, que os relatórios consultados continham vários resultados analíticos de outros agentes químicos descritos na Resolução CONAMA 344/04 (CONAMA, 2004), particularmente os metais e substâncias orgânicas, no entanto esses dados não foram apresentados pois raramente as concentrações ultrapassaram a classificação de nivel I da Resolução, ou não se apresentaram em quantidades suficientes para causar os efeitos tóxicos apresentados na Tabela 1.

Devido ao conhecimento da ausência de uma correlação evidente entre os efeitos tóxicos e as quantidades dos agentes químicos, poder-se-ia esperar que a mortalidade de Leptocheirus plumulosus e Tiburonella viscana fosse menor que $15 \%$, conforme requerido para os controles experimentais na norma NBR-15638 (ABNT, 2008). Entretanto, essa condição torna-se difícil de ser obtida com amostras de sedimento naturais que, além da possibilidade de conterem poluentes não mensurados, podem possuir características peculiares que afetam a sobrevivência dos organismos-teste porém não de modo significativo. Diante desse fato, os resultados dos ensaios ecotoxicológicos são discutidos com base em uma análise comparativa entre os dois anfípodos.

A Tabela 1 mostra que a média da mortalidade, nos diversos pontos amostrais, situou-se dentro do esperado para um ambiente pouco perturbado em termos ecotoxicológicos, tanto para Leptocheirus plumulosus (entre 11,8 e 26,8\%) como para Tiburonella viscana (entre 22 e 37\%). Além disso, nesta tabela é possível observar que nos pontos 1 a 4 a intensidade da mortalidade foi semelhante para os dois anfípodos, enquanto nos pontos 5 a 9 a intensidade aumentou para Tiburonella viscana. Aparentemente, o aumento da intesidade do efeito tóxico para esse anfípodo está acompanhada pelo aumento da proporção de silte+argila nas amostras, aspecto esse que será discutido mais adiante.

Outro aspecto abordado nesse estudo diz respeito a assunto polêmico entre os pesquisadores brasileiros, ou seja, a variação da resposta nas replicatas utilizadas nos ensaios com anfípodos. Para avaliar essa variação foi utilizada a sobrevivência como resposta, visto que apresenta valores mais constantes e de melhor quantificação. A verificação dos resultados das 108 amostras analisadas apontou que a média do Coeficiente de Variação (C.V.) entre as replicatas foi de $22 \%$ para ambos os anfípodos. Esses resultados concordam com os de Long et al. (1990) que observaram C.V. $=21,4 \%$ em replicatas de ensaios com sedimento e o anfípodo Rhepoxynius abronius.

Ao mesmo tempo, utilizou-se esse universo amostral (excluindo os ensaios com zero e 100\% de sobrevivência) para avaliar a correspondência entre a intensidade do efeito tóxico e o coeficiente de variação resultante. A Figura 2 mostra, para ambos os anfípodos, que quanto maior a sobrevivência menor é o coeficiente de variação obtido, fato que concorda com os estudos de variação interlaboratorial descritos pela USEPA(1994). No entanto, chama a atenção que nos ensaios com Tiburonella viscana (Figura $2 \mathrm{~b}$ ) o coeficiente de determinação $\left(\mathrm{r}^{2}\right)$ indicou a explicação de apenas 33\% das variações associdadas entre os coeficientes de variação e a sobrevivência, enquanto que nos ensaios com Leptocheirus plumulosus 58\% dessas variações tenham sido explicadas (Figura 2a). A princípio, seria esperado que os dois anfípodos apresentassem maior concordância dos coeficientes de determinação, com explicação próxima àquela obtida na correlação com Leptocheirus plumulosus.

Tabela 1 - Resultados (média e amplitude) dos ensaios ecotoxicológicos com anfipodos e das análises granulométricas e químicas, das 108 amostras de sedimento analisadas.

\begin{tabular}{|c|c|c|c|c|c|c|c|c|}
\hline \multirow{2}{*}{$\begin{array}{c}\text { Ponto de } \\
\text { amostragem }\end{array}$} & \multicolumn{2}{|c|}{ Mortalidade dos anfípodos (\%) } & \multicolumn{4}{|c|}{ Classificação dos grânulos (\%) } & \multirow{2}{*}{$\begin{array}{l}\text { Carbono orgânico } \\
\text { total }(\%)\end{array}$} & \multirow{2}{*}{$\begin{array}{l}\text { Nitrogênio Kjeldah } \\
\left(\text { g.kg }{ }^{-1}\right)\end{array}$} \\
\hline & $\begin{array}{c}\text { Leptocheirus } \\
\text { plumulosus }\end{array}$ & $\begin{array}{c}\text { Tiburonella } \\
\text { viscana }\end{array}$ & Areia & Silte & Argila & $\begin{array}{l}\text { Silte }+ \\
\text { Argila }\end{array}$ & & \\
\hline 1 & $\begin{array}{c}15,4 \\
\text { (zero-46) }\end{array}$ & $\begin{array}{r}22,0 \\
(12-35)\end{array}$ & $\begin{array}{c}93,6 \\
(87,7-97,5)\end{array}$ & $\begin{array}{c}3,8 \\
(1,6-9,0)\end{array}$ & $\begin{array}{c}2,1 \\
(0,8-4,2)\end{array}$ & $\begin{array}{c}5,9 \\
(2,6-11,8)\end{array}$ & $\begin{array}{c}0,5 \\
\text { (n.d. - } 1,2 \text { ) }\end{array}$ & $\begin{array}{c}1,0 \\
(0,1-4,8)\end{array}$ \\
\hline 2 & $\begin{array}{c}17,5 \\
\text { (zero-42) }\end{array}$ & $\begin{array}{r}25,8 \\
(10-45)\end{array}$ & $\begin{array}{c}84,7 \\
(53,2-95,9)\end{array}$ & $\begin{array}{c}12,5 \\
(1,5-44,3)\end{array}$ & $\begin{array}{c}2,3 \\
(0,4-8,2)\end{array}$ & $\begin{array}{c}14,8 \\
(2,5-47,0)\end{array}$ & $\begin{array}{c}0,4 \\
\text { (n.d.-0,2) }\end{array}$ & $\begin{array}{c}0,7 \\
(0,05-3,1)\end{array}$ \\
\hline 3 & $\begin{array}{l}21,9 \\
(8-52)\end{array}$ & $\begin{array}{r}26,0 \\
(8-58)\end{array}$ & $\begin{array}{c}65,6 \\
(27,3-93,7)\end{array}$ & $\begin{array}{c}31,0 \\
(5,3-68,6)\end{array}$ & $\begin{array}{c}3,3 \\
(1,3-8,1)\end{array}$ & $\begin{array}{c}34,3 \\
(7,1-72,2)\end{array}$ & $\begin{array}{l}2,7 \\
(0,4-5,6)\end{array}$ & $\begin{array}{c}1,1 \\
(0,3-2,7)\end{array}$ \\
\hline 4 & $\begin{array}{c}26,0 \\
(14-44)\end{array}$ & $\begin{array}{c}29,4 \\
(8-50)\end{array}$ & $\begin{array}{c}90,2 \\
(85,6-93,5)\end{array}$ & $\begin{array}{c}4,7 \\
(2,2-9,0)\end{array}$ & $\begin{array}{c}4,6 \\
(1,4-8,3)\end{array}$ & $\begin{array}{c}9,3 \\
(5,7-14,1)\end{array}$ & $\begin{array}{c}0,7 \\
\text { (n.d.- } 1,1 \text { ) }\end{array}$ & $\begin{array}{c}0,9 \\
(0,02-5,3)\end{array}$ \\
\hline 5 & $\begin{array}{l}18,3 \\
(2-38)\end{array}$ & $\begin{array}{r}33,6 \\
(10-62)\end{array}$ & $\begin{array}{c}44,8 \\
(28,7-78,7)\end{array}$ & $\begin{array}{c}49,7 \\
(19,9-66,4)\end{array}$ & $\begin{array}{c}4,8 \\
(1,4-10,3)\end{array}$ & $\begin{array}{c}54,5 \\
(21,3-68,6)\end{array}$ & $\begin{array}{c}2,5 \\
\text { (n.d.-4,9) }\end{array}$ & $\begin{array}{c}1,7 \\
(0,2-5,3)\end{array}$ \\
\hline 6 & $\begin{array}{l}26,8 \\
(8-66)\end{array}$ & $\begin{array}{r}37,0 \\
(12-100)\end{array}$ & $\begin{array}{c}44,4 \\
(17,2-58,2)\end{array}$ & $\begin{array}{c}47,9 \\
(23,6-62,2)\end{array}$ & $\begin{array}{c}7,3 \\
(3,3-23,1)\end{array}$ & $\begin{array}{c}55,2 \\
(25,9-81,9)\end{array}$ & $\begin{array}{l}3,1 \\
(1,9-5,8)\end{array}$ & $\begin{array}{c}1,9 \\
(0,1-4,5)\end{array}$ \\
\hline 7 & $\begin{array}{c}13,6 \\
\text { (zero- } 28)\end{array}$ & $\begin{array}{c}31,1 \\
(5-54)\end{array}$ & $\begin{array}{c}68,2 \\
(48,8-81,7)\end{array}$ & $\begin{array}{c}26,4 \\
(14,0-38,9)\end{array}$ & $\begin{array}{c}5,0 \\
(2,0-18,5)\end{array}$ & $\begin{array}{c}31,4 \\
(18,5-49,3)\end{array}$ & $\begin{array}{c}1,3 \\
\text { (n.d.-2,4) }\end{array}$ & $\begin{array}{c}1,4 \\
(0,4-6,8)\end{array}$ \\
\hline 8 & $\begin{array}{l}13,7 \\
(2-36)\end{array}$ & $\begin{array}{r}30,6 \\
\text { (zero-60) }\end{array}$ & $\begin{array}{c}63,6 \\
(33,0-77,7)\end{array}$ & $\begin{array}{c}29,0 \\
(15,5-61,9)\end{array}$ & $\begin{array}{c}7,0 \\
(1,6-20,1)\end{array}$ & $\begin{array}{c}36,0 \\
(22,1-67,0)\end{array}$ & $\begin{array}{l}1,1 \\
(0,3-1,6)\end{array}$ & $\begin{array}{c}1,0 \\
(0,24-5,2)\end{array}$ \\
\hline 9 & $\begin{array}{c}11,8 \\
\text { (zero- } 28)\end{array}$ & $\begin{array}{r}30,6 \\
(5-42)\end{array}$ & $\begin{array}{c}84,6 \\
(42,2-92,9)\end{array}$ & $\begin{array}{c}11,4 \\
(4,5-44,5)\end{array}$ & $\begin{array}{c}3,6 \\
(1,2-4,6)\end{array}$ & $\begin{array}{c}15,0 \\
(6,8-56,8)\end{array}$ & $\begin{array}{c}0,6 \\
\text { (n.d-1,5) }\end{array}$ & $\begin{array}{c}0,9 \\
(0,1-2,7)\end{array}$ \\
\hline
\end{tabular}

n.d.= não detectado. 
Com a finalidade de averiguar as possíveis causas das correlações diferenciadas para os dois anfípodos, efetuou-se a análise de regressão apenas com os resultados de mortalidade e das análises químicas e físicas descritas na Tabela 1. Esse tipo de análise é conveniente visto que as amostras de sedimento analisadas não continham poluentes em quantidades suficientes para causar um efeito tóxico ou, ainda, que as substâncias estavam abaixo do nível I da Resolução CONAMA 344/04 (CONAMA, 2004). Na Tabela 2 são apresentados os coeficientes de determinação resultantes, os quais indicam que os resultados dos ensaios com Leptocheirus plumulosus não se correlacionaram, significativamente, com as análises químicas e físicas. No que se refere aos resultados dos ensaios com Tiburonella viscana verificou-se existir correlações, significativas, entre a mortalidade e os resultados das análises granulométricas e de nitrogênio Kjeldahl.

Aparentemente, a correlação entre os teores de nitrogênio Kjeldahl e a mortalidade de Tiburonella viscana está associada à presença de silte e argila, os quais propiciam uma maior adsorção do nitrogênio ao sedimento. Essa inferência foi confirmada pela análise de regressão múltipla da mortalidade/ silte+argila/N-Kjeldahl que não foi significativa $(p>0,05) \mathrm{com}$ $\mathrm{r}^{2}=0,61$, ou seja, praticamente não melhorou a correlação entre mortalidade/silte+argila $\left(\mathrm{r}^{2}=0,59, \mathrm{p}<0,02\right)$ descrita na Tabela 2.

Por outro lado, a tentativa de correlacionar a mortalidade da Tiburonella viscana e a concentração de nitrogênio Kjeldahl torna-se difícil, visto que, em termos ecotoxicológicos, a amônia
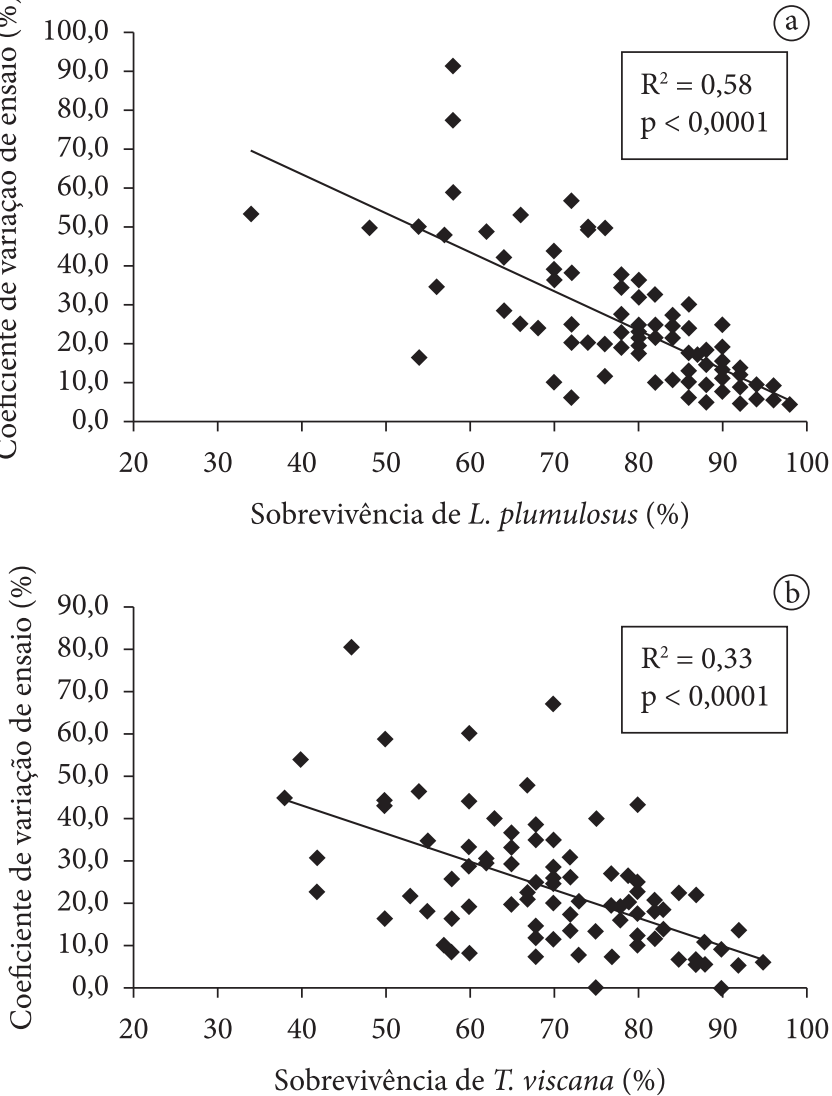

Figura 2 - Regressão linear entre a sobrevivência dos anfípodos e o respectivo coeficiente de variação nos ensaios com a) Leptocheirus plumulosus; e b) Tiburonella viscana. não ionizada é sabidamente a principal forma nitrogenada que causa os efeitos tóxicos a organismos aquáticos (Russo, 1985). Assim, somente considerando haver uma proporção idêntica de nitrogênio Kjeldahl e amônia não ionizada em todas as amostras de sedimento, poder-se-ia supor que a correlação com o efeito tóxico para Tiburonella viscana tenha sido ocasionada pela maior sensibilidade desse anfípodo à amônia. Essa suposição é pertinente quando se compara a sensibilidade dos dois anfípodos em outros estudos. Segundo a USEPA (1994) Leptocheirus plumulosus não sofre efeitos tóxicos em concentrações $<0,8 \mathrm{mg} \cdot \mathrm{L}^{-1}$ de amônia não ionizada, enquanto que concentrações $<0,3 \mathrm{mg} \cdot \mathrm{L}^{-1}$ dessa substância são desejáveis para evitar um efeito tóxico para Tiburonella viscana (comunicação pessoal da Biol. Regina Sawaia Sáfadi).

Torna-se oportuno ressaltar que a amônia não ionizada deve ser sempre quantificada em ensaios ecotoxicológicos com sedimento integral e anfípodos, particularmente aquela encontrada na água intersticial, visto que esse agente químico pode se tornar um interferente analítico nos resultados obtidos, isto é, os efeitos tóxicos podem ser produzidos por quantidades naturais de amônia não ionizada ao invés de outros poluentes de interesse. Por esse motivo a norma ABNT-NBR 15638 (ABNT, 2008) recomenda, explicitamente, a análise de amônia na água intersticial do ensaio ecotoxicológico com sedimento integral.

Para simplificar a discussão a seguir, sobre as correlações entre a mortalidade dos anfípodos e a análise granulométrica descritas na Tabela 2, foram utilizados somente os dados de mortalidade e a fração silte+argila, também conhecida como finos. Essa abordagem se justifica pois a fração silte+argila agrega as porções individuais dessas frações e, também, pelo fato dos dados da fração areia se referir ao percentual complementar da fração silte+argila. No tocante a essas correlações, somente a mortalidade de Tiburonella viscana apresenta correlações significativas com as variáveis granulométricas. Essa constatação comprova as observações previamente mencionadas sobre o aumento da mortalidade desse anfípodo em função da proporção de silte+argila nas amostras de sedimento dos pontos 5 a 9 (Tabela 1).

A ausência de correlação entre a mortalidade de Leptocheirus plumulosus e a fração silte+argila concorda com o descrito por vários autores citados pela USEPA (1994), isto é, a mortalidade desse organismo não é afetada pelas variações extremas da granulometria dos sedimentos. Quanto a Tiburonella viscana, os resultados da Tabela 1 contradizem os achados de Melo \&

Tabela 2 - Coeficientes de determinação $\left(\mathrm{r}^{2}\right)$ entre a mortalidade dos anfípodos e as variáveis químicas e físicas do sedimento.

\begin{tabular}{lcc}
\hline \multirow{2}{*}{ Variáveis } & \multicolumn{2}{c}{ Coeficiente de determinação $\left(\mathrm{r}^{2}\right)$} \\
\cline { 2 - 3 } & $\begin{array}{c}\text { Mortalidade de } \\
\text { Leptocheirus plumulosus }\end{array}$ & $\begin{array}{c}\text { Mortalidade de } \\
\text { Tiburonella viscana }\end{array}$ \\
\hline areia & 0,05 & $0,59^{*}$ \\
silte & 0,07 & $0,53^{*}$ \\
argila & 0,06 & $0,70^{*}$ \\
silte + argila & 0,07 & $0,59^{*}$ \\
carbono orgânico total & 0,28 & 0,34 \\
nitrogênio Kjeldahl & 0,10 & $0,54^{*}$ \\
\hline
\end{tabular}

* correlação significativa $(\mathrm{p}<0,05)$. 
Nipper (2007) que sugerem a sobrevivência desse anfípodo não ser afetada em sedimentos com proporção granulométrica até $60 \%$ de silte+argila.

Para esclarecer essa divergência foram selecionados alguns estudos realizados com Tiburonella viscana, para os quais efetuou-se a análise de regressão com os resultados de mortalidade/sobrevivência e da fração silte+argila. Na Tabela 3 , notou-se que a correlação entre essas variáveis também ocorreu em outros estudos, sendo que nos trabalhos de Choueri et al. (2009) e Melo \& Nipper (2007) houve uma explicação significativa (77 e $57 \%$, respectivamente), semelhantes àquela obtida no presente estudo (59\%). Além das correlações, o ponto comum dos três estudos mencionados reside no fato de não haver evidências categóricas de que os efeitos tóxicos tenham sido provocados por agentes químicos em concentrações elevadas.

Sob o mesmo ponto de vista, na Tabela 3 são apresentados outros trabalhos nos quais os coeficientes de determinação foram menores, porém ainda significativos. Na pesquisa de Abessa (2002) o coeficiente de determinação indica que $28 \%$ da variação dos efeitos tóxicos para Tiburonella viscana foi influenciada pela fração silte+argila e, segundo o autor, as demais variações foram explicadas por outros fatores tal como a quantidade de mercúrio. No artigo de Sousa et al. (2007) constatou-se uma a correlação significativa $\left(r^{2}=0,36\right)$ entre os efeitos tóxico para Tiburonella viscana e a proporção de silte+argila, no entanto, os referidos autores não apresentaram outras análises químicas que permitissem explicar as demais variações na ecotoxicidade das amostras.

Para os demais estudos apresentados na Tabela 3 (Cesar et al., 2006; Moreira, 2009), a ausência de correlações significativas entre os resultados dos ensaios com Tiburonella viscana e a fração silte+argila se justifica pela baixa variação dessa fração nas amostras de sedimento, isto é, com média inferior a $10 \%$ e amplitude entre 24 e $0,08 \%$. Vale destacar a pesquisa de Morais et al. (2009), os quais realizaram ensaios ecotoxicológicos com Tiburonella viscana e granulométricos com amostras coletadas nos mesmos pontos pesquisados por Choueri et al. (2009). Nesses dois estudos seria razoável esperar que a intensidade dos efeitos tóxicos fosse semelhante, no entanto, Morais et al. (2009) verificaram uma sobrevivência elevada do anfípodo em oposição ao detectado por Choueri et al. (2009). A análise comparativa desses estudos evidencia que, em geral, a sobrevivência elevada de Tiburonella viscana foi associada à proporção reduzida de silte+argila nas amostras de sedimento no trabalho de Morais et al. (2009).

Em função das discussões antecedentes, torna-se evidente de que os resultados do ensaio ecotoxicológico com Tiburonella viscana expressam, além dos efeitos tóxicos de agentes químicos, os efeitos adversos da proporção de silte+argila nos sedimentos ensaiados. Esse fato também foi constatado por DeWitt et al. (1988) em ensaios com o anfípodo Rhepoxynius abronius. Esses autores verificaram que embora houvesse correlação entre os agentes químicos e a sobrevivência do Rhepoxynius abronius em sedimentos não contaminados, as quantidades dos poluentes não eram suficientes para causar a mortalidade dos anfípodos, mas sim o tamanho dos grânulos dos sedimentos. Por tal motivo esses autores elaboraram equações matemáticas, que são recomendadas pela USEPA (1994), para tentar distinguir a mortalidade do Rhepoxynius abronius associada às proporções de silte+argila. Para se ter uma ideia, com o uso dessas equações Ferraro \& Cole (2002) detectaram $13 \%$ de resultados com a suspeita de interferência da fração silte+argila, enquanto DeWitt et al. (1988) encontraram 65\% dos resultados com a mesma suspeita.

Com a finalidade de estimar a proporção de silte+argila que não causa efeitos adversos, e seguindo os mesmos princípios de DeWitt et al. (1988), selecionou-se aqueles estudos com Tiburonella viscana nos quais não houve evidências de contaminação química. Assim, foi efetuada a análise de regressão utilizado-se os dados das pesquisas de Melo \& Nipper (2007), Choueri et al. (2009) e do presente artigo (Tabela 1), totalizando 23 pares de resultados ecotoxicológicos e granulométricos. $\mathrm{O}$ resultado da análise de regressão está demonstrado na Figura 3.

Os limites de previsão, apresentados na Figura 3, são aqueles dentro dos quais se espera localizar $95 \%$ das observações futuras e independentes do conjunto de dados utilizados (Draper $\&$ Smith, 1981). Assim, tomando como base o limite inferior de previsão, é possível verificar que a mortalidade admitida nos ensaios com anfípodos (15\%) corresponde à proporção de $33 \%$ de silte + argila em uma amostra de sedimento, conforme

Tabela 3 - Coeficientes de determinação e nível de significância entre a mortalidade/sobrevivência de Tiburonella viscana e a fração silte+argila, em diversos estudos.

\begin{tabular}{lc}
\hline Estudo analisado & Coeficiente de determinação $\left(\mathrm{r}^{2}\right)$ \\
\hline Presente trabalho & $0,59(\mathrm{p}=0,02)^{*}$ \\
Choueri et al. (2009) & $0,77(\mathrm{p}=0,05)$ \\
Melo \& Nipper (2007) & $0,57(\mathrm{p}=0,02)$ \\
Abessa (2002) & $0,28(\mathrm{p}=0,004)$ \\
Sousa et al. $(2007)$ & $0,36(\mathrm{p}=0,02)$ \\
Morais et al. $(2009)$ & $0,42(\mathrm{p}=0,23)$ \\
Cesar et al. (2006) & $0,16(\mathrm{p}=0,43)$ \\
& $0,34(\mathrm{p}=0,08)$ \\
Moreira (2009) & $0,15(\mathrm{p}=0,52)$ \\
& $0,004(\mathrm{p}=0,92)$ \\
\hline
\end{tabular}

* nível de significância da correlação.

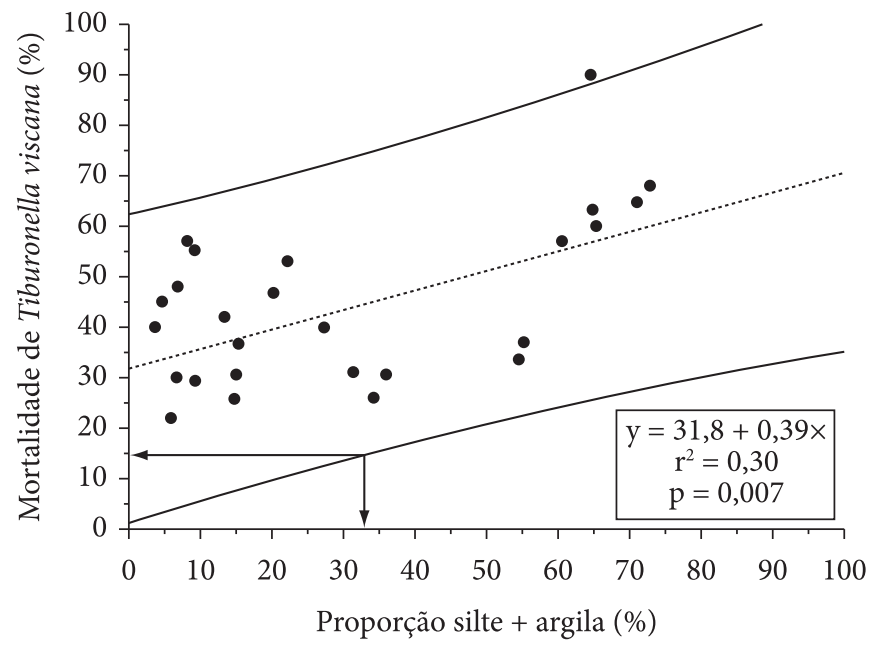

Figura 3 - Reta de regressão (linha tracejada) e limites de previsão (linhas sólidas) entre a proporção de silte+argila do sedimento e a mortalidade de Tiburonella viscana. 
mostrado na Figura 3. Deste modo, é esperado que proporções de silte+argila maiores que $33 \%$ causem efeitos tóxicos significativos a Tiburonella viscana, isoladamente ou pela interação com os agentes químicos presentes. Essa condição é fundamental ser observada para evitar a ocorrência de resultados falsos-positivos, nos ensaios com Tiburonella viscana, particularmente quando se utiliza o "teste t" para verificar a existência de efeitos tóxicos significativos.

Vale destacar que, em função do coeficiente de determinação obtido, os limites de previsão apresentados na Figura 3 admitem para a proporção de $33 \%$ de silte+argila uma variação entre 15 e 70\% de mortalidade da Tiburonella viscana. Embora o coeficiente de determinação seja reduzido $\left(r^{2}=0,30\right)$ o nível de significância $(\mathrm{p}=0,007)$ é bastante expressivo, fato que permite efetuar projeções na ausência de pesquisa mais direcionada sobre o assunto. Ressalta-se que no artigo de DeWitt et al. (1988) foi demonstrado um coeficiente de determinação muito próximo para o anfípodo Rhepoxynius abronius $\left(\mathrm{r}^{2}=0,29, \mathrm{p}<0,001\right)$, embora com um universo de dados pareados bastante maior $(n=315)$ do que o utilizado no presente estudo $(n=23)$.

Portanto, os pesquisadores brasileiros devem estar atentos quando da obtenção de resultados nos ensaios com amostras de sedimento e o anfípodo Tiburonella viscana. Como demonstrado, quando a fração silte+argila excede $33 \%$ em amostra de sedimento é duvidoso atribuir os efeitos tóxicos exclusivamente às concentrações de agentes químicos. Esse tipo de ocorrência é particularmente importante em sedimentos pouco poluídos como os apresentados nesse estudo, o que concorda com as pesquisas de DeWitt et al. (1988) e Long et al. (1990) para o anfípodo Rhepoxynius abronius. Assim, quando há a constatação de efeitos tóxicos, também deve-se efetuar uma análise cuidadosa dos poluentes suspeitos, com base em dados da literatura, para verificar se as quantidades encontradas são suficientes para causar o efeito detectado.

Diante desses fatos, quando se desconhece previamente a proporção de silte+argila do sedimento, há a necessidade da análise granulométrica antes do início do ensaio. Desse modo, caso a fração silte+argila seja $>33 \%$ deve-se utilizar um controle experimental adicional que contenha a mesma proporção dessa fração. Uma alternativa, quando se desconhece a composição granulométrica dos sedimento a ser ensaiado, seria o uso da análise estatística "t por bioequivalência". Segundo Buratini \& Bertoletti (2008), essa análise estatística permite detectar se as médias obtidas nos grupos experimentais diferem do grupo controle de modo significativo do ponto de vista estatístico e, principalmente, do biológico. Torna-se oportuno ressaltar que a significância biológica é bastante influenciada, entre outras variáveis, pela variabilidade dos efeitos tóxicos nas replicatas do ensaio, como demonstrado na Figura 2b. Assim, na análise estatística "t por bioequivalência" são utilizadas as constantes de proporcionalidade, expressas em porcentagem, as quais já foram estabelecidas em 0,80 para a sobrevivência nos ensaios com Leptocheirus plumulosus (comunicação pessoal de Valéria A. Prósperi) e de 0,69 nos ensaios com Tiburonella viscana (comunicação pessoal de Denis M. S. Abessa). Nos termos do presente estudo essas constantes de proporcionalidade correspondem a 20 e $31 \%$ de mortalidade esperada para Leptocheirus plumulosus e Tiburonella viscana, respectivamente.
Particularmente quanto aos ensaios com Tiburonella viscana, no presente estudo foram efetuados os cálculos estatísticos com 99 ensaios (dados não apresentados) para verificar o valor da constante de proporcionalidade, sendo que foi confirmado o porcentual de 0,69. Deve-se notar que essa constante de proporcionalidade foi calculada englobando a variabilidade do efeito adverso causado pela fração silte+argila nas replicatas dos ensaios (Figura 2b). Desse modo, o uso da estatística "t por bioequivalência" mostra-se apropriado, tanto no presente como em outros trabalhos, para avaliar a ocorrência do efeito tóxico significativo devido, exclusivamente, aos agentes químicos presentes no sedimento.

Em função do exposto, a escolha do anfípodo para os ensaios com sedimento integral de ambientes marinhos deve levar em consideração as particularidades experimentais exigidas pelos organismos-teste. No caso de utilização do Leptocheirus plumulosus a exigência mais importante é o conhecimento do teor de nitrogênio amoniacal, sendo que a característica eurialina desses organismos e a possibilidade de cultivo, em laboratório, o tornam bastante conveniente.

No caso do uso da Tiburonella viscana, além do nitrogênio amoniacal, é fundamental que se conheça a proporção de silte+argila nas amostras, a qual exigirá procedimento analítico diferenciado ou a utilização de análise estatística apropriada. Adicionalmente, é necessário considerar que a Tiburonella viscana trata-se de um organismo estenoalino e, também, que até o momento não foram estabelecidos procedimentos para o seu cultivo em laboratório. Torna-se oportuno mencionar que, embora vários pesquisadores suspeitem da sensibilidade oscilante da Tiburonella viscana, devido às coletas sazonais desses organismos, no presente estudo tal suspeita não se comprovou, tendo como base os ensaios de sensibilidade efetuados com os diferentes lotes desse organismo-teste.

\section{REFERÊNCIAS BIBLIOGRÁFICAS}

ABESSA, D. M. S., 2002, Avaliação da qualidade de sedimentos do sistema estuarino de Santos, SP, Brasil. Tese (Doutorado)Instituto Oceanográfico, USP, 295p.

ABNT, Associação Brasileira de Normas Técnicas, 2008, Qualidade de água - Determinação da toxicidade aguda de sedimentos marinhos ou estuarinos com anfipodos. Rio de Janeiro, 19p.

BURATINI, S. V. \& BERTOLETTI, E., 2008, Análise estatística. In: P. A. Zagatto \& E. Bertoletti (ed.). Ecotoxicologia aquática: princípios e aplicações. RIMA, São Carlos, p.221-249.

CESAR, A., PEREIRA, C. D. S., SANTOS, A. R., ABESSA, D. M. S., FERNÁNDEZ, N., CHOUERI, R. B. \& DelVALLS, T. A., 2006, Ecotoxicological assessment of sediments from the Santos and São Vicente estuarine system - Brazil. Braz. J. Oceanogr., 54:55-63. doi: 10.1590/S1679-87592006000100005.

CHOUERI, R. B., CESAR, A., TORRES, R. J., ABESSA, D. M. S., MORAIS, R. D., PEREIRA, C. D. S, NASCIMENTO, M. R. L., MOZETO, A. A., RIBA, I. \& DelVALLS, T. A., 2009, Integrated sediment quality assessment in Paranaguá Estuarine System, Southern Brazil. Ecotoxicol. and Environ. Saf., 72:1824-1831. doi: 10.1016/j.ecoenv.2008.12.005.

CONAMA, Conselho Nacional do Meio Ambiente, 2004, Resolução CONAMA 344 de 25 mar. 2004. Diário Oficial da União, Brasília, DF, 07 maio 2004. Retificada em 28 maio 2004. 
DeWITT, T. H., DITSWORTH, G. R. \& SWARTZ, R. C., 1988, Effects of natural sediment features on survival of the Phoxocephalid amphipod, Rhepoxynius abronius. Mar. Environ. Res., 25:99-124. doi:10.1016/0141-1136(88)90006-2.

DRAPER, N. R. \& SMITH, H., 1981, Applied regression analysis. John Wiley \& Sons, Inc., New York, 709p.

FERRARO, S. P. \& COLE, F. A., 2002, A field validation of two sediment-amphipod toxicity tests. Environ. Toxicol. Chem., 21:1423-1437. doi: 10.1002/etc.5620210714.

INGERSOLL, C. G., ANKLEY, G. T., BAUDO, R., BURTON, G. A., LICK, W., LUOMA, S. N., MacDONALD, D. D., REYNOLDSON, T. B., SOLOMON, K. R., SWARTZ, R. C. \& WARREN-HICKS, W. J., 1997, Workgroup summary report on uncertainty evaluation of measurement endpoints used in sediment ecological risk assessment. In: C. G. Ingersoll, T. Dillon \& G. R. Biddinger (ed.), Ecological risk assessment of contaminated sediments. SETAC Press, Pensacola, FL, p.297-352.

LONG, E. R., BUCHMAN, M. F., BAY, S. M., BRETELER, R. J., CARR, R. S., CHAPMAN, P. M., HOSE, J. E., LISSNER, A. L., SCOTT, J. \& WOLFE, D. A., 1990, Comparative evaluation of five toxicity tests with sediments from San Francisco bay and Tomales bay, California. Envirn. Toxicol. Chem., 9:1193-1214. doi: 10.1002/etc.5620090910.

MELO, S. L. R. \&ABESSA, D. M. S., 2002, Testes de toxicidade com sedimentos marinhos utilizando anfipodos. In: I. A. Nascimento, E. C. P. M. Sousa \& M. Nipper. (ed), Métodos em ecotoxicologia marinha. Artes Graf. Ind. Ltda., São Paulo, p.163-178.

MELO, S. L. R. \& NIPPER, M., 2007, Sediment toxicity tests using the burrowing amphipod Tiburonella viscana (Amphipoda:
Platyischnopidae). Ecotoxicol. Environ. Saf. 66:412-420. doi: 10.1016/j.ecoenv.2005.12.003.

MORAIS, R. D., MOREIRA, L. B., MARANHO, L. A., ABESSA, D. M. S., CESAR, A. \& MACHADO, E. C. 2009. Avaliação da toxicidade de sedimentos no complexo estuarino de Paranaguá. In: E. B. Boldrini \& E. V. Paula. Gestão ambiental portuária: subsídios para o licenciamento das dragagens. ADEMADAN, Curitiba, PR, p.300-307.

MOREIRA, L. B., 2009, Avaliação da toxicidade dos sedimentos e macrofauna bentônica em áreas portuárias: Porto do Mucuripe e terminal portuário do Pecém (CE), e Porto de Santos (SP). Dissertação (Mestrado)-Instituto de Ciências do Mar, UFC, $134 p$.

PRÓSPERI, V. A. \& NASCIMENTO, I. A., 2008, Avaliação ecotoxicológica de ambientes marinhos e estuarinos. 2. ed. In: Zagatto, P. A. \& Bertoletti, E. (ed.), Ecotoxicologia aquática: princípios e aplicações. RIMA, São Carlos, p.269-292.

RUSSO, R. C., 1985, Ammonia, nitrite, and nitrate. In: G. M. Rand \& S. R. Petrocelli (ed.). Fundamentals of aquatic toxicology. Hemisphere Co., Cambridge, p.455-471.

SOUSA, E. C. P. M., ABESSA, D. M. S., RACHID, B. R. F., GASPARRO, M. R. \& ZARONI, L. P., 2007, Ecotoxicological assessment of sediments from the port of Santos and the disposal sites of dredged material. Braz. J. Oceanogr., 55:75-81. doi: 10.1590/S1679-87592007000200001.

USEPA, U.S. Environment Protection Agency, 1994, Methods for assessing the toxicity of sediment-associated contaminants with estuarine and marine amphipods. Office of Research and Development, Narragansett, RI, 140p. EPA/600/R-94/025. 
